\title{
Somatoform disorders and rheumatic diseases: from DSM-IV to DSM-V
}

\author{
A. Alciati ${ }^{1}$, F. Atzeni ${ }^{2}$, P. Sgiarovello², P. Sarzi-Puttini ${ }^{2}$ \\ ${ }^{1}$ Hermanas Hospitalarias, FoRiPsi, Dipartimento di Neuroscience Cliniche, Villa San Benedetto Menni, \\ Albese con Cassano, Como; \\ 2U.O. di Reumatologia, Ospedale Universitario L. Sacco, Milan, Italy
}

\begin{abstract}
SUMMARY
Medically unexplained symptoms are considered 'somatoform disorders' in the fourth edition of the Diagnostic and Statistical Manual of Mental Disorders (DSM-IV). The introduction of this nosographic category has been helpful in drawing attention to a previously neglected area, but has not been successful in promoting an understanding of the disorders' biological basis and treatment implications, probably because of a series of diagnostic shortcomings.

The newly proposed DSM-V diagnostic criteria try to overcome the limitations of the DSM-IV definition, which was organised centrally around the concept of medically unexplained symptoms, by emphasising the extent to which a patient's thoughts, feelings and behaviours concerning their somatic symptoms are disproportionate or excessive. This change is supported by a growing body of evidence showing that psychological and behavioural features play a major role in causing patient disability and maintaining high level of health care use. Pain disorders is the sub-category of DSM-IV somatoform disorders that most closely resembles fibromyalgia. Regardless of the diagnostic changes recently brought about by DSM-V, neuroimaging studies have identified important components of the mental processes associated with a DSM- IV diagnosis of pain disorder.
\end{abstract}

Key words: Pain disorders, Statistical manual of mental disorders, Fibromyalgia.

Reumatismo, 2014; 66 (1): 98-102

\section{INTRODUCTION}

M ore than $80 \%$ of the general population report somatic symptoms that are not attributable to a clearly defined medical condition during the previous seven days (1). These findings indicate that somatic symptoms are ubiquitous and do not per se justify a diagnosis, especially a diagnosis of mental disorder. However, a subgroup of the people reporting somatic symptoms have long-lasting complaints, make considerable use of healthcare services, and appear to be seriously disabled, thus making it necessary to find other factors for identifying them.

Corresponding author: Alessandra Alciati Hermanas Hospitalarias - FoRiPsi Dipartimento di Neuroscience Cliniche

Villa San Benedetto Menn Albese con Cassano, Como, Italy E-mail: alessandra.alciati@libero.it

\section{SOMATOFORM DISORDERS FROM DSM-III TO DSM-IV-TR}

The disorders characterised by medically unexplained symptoms lie in an indefinite area between medicine (which classifies them as functional somatic syndromes) and psychiatry (2) which, from the third edition of Diagnostic and Statistical Manual of Mental Disorders (DSM III) (3) published in 1980, classified as them as somatoform disorders. In clinical practice, only a few patients with somatic complaints are examined by a psychiatrist; the majority are seen by a general practitioner or by a specialist who treats the symptom or function that leads to the distress. It is known that somatic symptoms not explained by a general medical condition account for almost half of the complaints reported by patients seeking primary and secondary care (4). The introduction of somatoform disorders as a diagnostic category represented an advance in clinical psychiatry because it replaced the previous ambiguous nosology based on diagnostically unreliable with a more precise and systematic classification. However, although this has been very 
helpful in drawing attention to a previously neglected area, it has not been successful in promoting an understanding of the disorders' biological basis and treatment, probably because of a series of shortcomings in the category itself and its specific sub-categories (5).

The diagnostic category of somatoform disorders has been considered speculative; ambiguous in its stated exclusion of general medical conditions; imprecise because of its overlaps with other psychiatric disorders such as depression and anxiety whose diagnostic definitions include somatic symptoms (5); and scarcely compatible with, or even unacceptable to many oriental cultures that do not share the West's dualistic view of mind and body (as suggested by its exclusion from the current DSM-based Chinese classification of mental diseases) (6).

The specific diagnostic sub-categories formulated in the Diagnostic and Statistical Manual of Mental Disorders-IV - Text Revision (DSM-IV-TR) (7) include a heterogeneous group of disorders united solely by their presentation with somatic symptoms (Tab. I). The main shortcoming of these sub-categories is that their considerable diagnostic overlaps make them confusing and of little clinical use, particularly in the non-psychiatric general medical settings primarily attended by such patients (8). It is not surprising that more than $90 \%$ of physicians report an overlap between somatisation disorder, pain disorder, hypochondriasis, and "somatoform disorder not otherwise specified" (9).

For patients with multiple medically unexplained symptoms, the DSM suggests a diagnosis of somatisation disorder (the core

Table I - Somatoform disorders: DSM-IV - TR codes and specific diagnostic sub-categories.

\begin{tabular}{|l|l|}
\hline 300.81 & Somatisation disorder \\
\hline 300.81 & Undifferentiated somatoform disorder \\
\hline 300.11 & Conversion disorder \\
\hline 307.80 & Pain disorder \\
\hline 300.7 & Hypochondriasis \\
\hline 300.7 & Body dysmorphic disorder \\
\hline 300.81 & $\begin{array}{l}\text { Somatoform disorder not otherwise } \\
\text { specified }\end{array}$ \\
\hline
\end{tabular}

sub-category of somatoform disorders) that is based on the number of unexplained medical symptoms, and requires four pain, two gastrointestinal, one sexual and one "pseudo-neurological" symptom. These criteria have been criticised as being too restrictive because they cover only a very small proportion $(0.1 \%)$ of subjects in the general population reporting multiple somatic symptoms requiring medical attention $(10 \%)(10)$.

The "undifferentiated somatoform disorder" (USD) sub-category describes a set of unexplained physical symptoms that do not cross the threshold of somatisation disorder, but the diagnostic criteria were so vaguely defined that they would be met by a large percentage of the general population (a reported primary prevalence of $27 \%$ ) (11). Somatisation disorder not otherwise specified is kept as a residual category.

This finding supported the need to reduce the number of somatic complaints required for a diagnosis of somatisation disorder (12). As temporal stability is one of the validation criteria of a psychiatric syndrome diagnosis, the hypothesis of reducing the number of somatic symptoms is supported by the evidence that spontaneous remission rates are high if patients report only one non- pain physical symptom whereas, in the presence of at least two somatic symptoms (or even one in the case of a pain symptom) (13), the syndrome seems to be stable over time (14).

Moreover, the classification of multiple somatic complaints as DSM mental disorders should consider integrating empirically derived psychological and behavioural characteristics with the formal diagnostic criteria (15) as it has been shown that these are the main predictors of symptom recurrence in chronic pain conditions (16), enable the detection of more stable syndromes (17), and can help distinguish medically explained and unexplained syndromes (18). Among patients with somatic syndromes, psychological factors can distinguish those with healthcare needs from those who can cope quite well without medical care. They can also explain significant aspects of disability even after controlling for the impact 
of somatic symptoms and depression (19). The most powerful psychological variable identifying people with somatic symptoms and increased healthcare use and/or serious disability is the avoidance of physical activities that accelerate the heart rate or cause sweating. Cognitive variables can also distinguish the more problematic sub-group of people suffering from somatic symptoms: catastrophising bodily sensations, making attributions to somatic illness, and worrying and ruminating about physical complaints all contribute to explaining the statistical impact of medically unexplained somatic symptoms on healthcare use and disability (19). A high level of use of health services implies excess annual healthcare costs that are comparable with those related to depression or anxiety disorders (20), and requires the development of strategies aimed at the early detection of somatising patients who might become costly users of health services (21).

\section{TOWARD DSM-V}

The fifth edition of the Diagnostic and Statistical Manual of Mental Disorders (DSMV) (22) introduces significant changes to the diagnostic criteria of somatoform disorders with the aim of eliminating overlaps and clarifying boundaries. The novelties of the DSM -V are so radical that they impose a parallel change in the name somatoform disorders to somatic symptom and related disorders (SSD). An SSD is characterised by one or more somatic symptoms lasting for at least six months that are very distressing or lead to significant disruption of functioning, as well as excessive and disproportionate thoughts, feelings and behaviours regarding those symptoms (Tab. II).

The main changes from the DSM-IV diagnosis of somatoform disorders are:

- all of the DSM-IV somatoform disorder sub-categories (somatisation disorder, pain disorder, undifferentiated somatoform disorder, hypochondriasis, etc.) have been deleted as most of the patients previously diagnosed with one of these now meet the diagnostic criteria of SSD;
Table II - DSM-V Criteria for somatic symptom disorder.

A. One or more somatic symptoms that are
distressing or result in significant disruption of daily
life.

B. Excessive thoughts, feelings, behaviours related to the somatic symptoms or associated health concerns, as manifested by at least one of the following:

1) Disproportionate and persistent thoughts about the seriousness of one's symptoms.

2) Persistently high level of anxiety about health or symptoms.

3) Excessive time and energy devoted to these symptoms or health concerns.

C. Although any one somatic symptom may not be continuously present, the state of being symptomatic is persistent (typically more than 6 months).

Reprinted with permission from the Diagnostic and Statistical Manual of Mental Disorders, Fifth Edition, (Copyright ${ }^{\odot}$ 2013). American Psychiatric Association. All rights reserved.

the SSD diagnosis does not require the somatic symptoms to be medically unexplained;

- the presence of maladaptive and disproportionate thoughts, feelings, and behaviours concerning the somatic symptoms becomes the central diagnostic feature of SSD, and the somatic symptom count required by some of the DSM-IV diagnostic categories has been deleted.

DSM-IV was organised centrally around the concept of medically unexplained symptoms, whereas the DSM-V criteria emphasise the extent to which a patient's thoughts, feelings and behaviours regarding their somatic symptoms are disproportionate or excessive.

\section{PAIN DISORDER}

Pain disorder is the sub-category of DSMIV somatoform disorders that most closely resembles fibromyalgia. DSM-IV defines pain disorder as the occurrence of one or more pain symptoms causing significant distress or impairment in several areas of functioning, with psychological factors being judged to play an important role in the onset, severity, exacerbation, or maintenance of the pain (Tab. III).

A number of studies have raised concerns regarding the validity of the distinction be- 
Table III - DSM-IV-TR Diagnostic Criteria for Pain Disorder.

\begin{tabular}{|l|}
\hline A. Pain in one or more anatomical sites is the \\
predominant focus of the clinical presentation and is \\
of sufficient severity to warrant clinical attention.
\end{tabular}

tween pain disorder and pain associated with other somatoform disorders made in DSM-IV (23). Aigner found a considerable overlap of somatoform disorder diagnoses in patients who also had a diagnosis of pain disorder, with $93 \%$ meeting the DSM-IV criteria for undifferentiated somatoform disorder and $10 \%$ meeting the criteria for somatisation disorder (24).

In line with these observations, more than one-third of surveyed physicians stated that the border between the diagnostic criteria for pain disorder and somatoform disorder not otherwise specified is "unclear" (9).

The perspective change introduced by DSM-V has also promoted a different approach to the disorders characterised by pain symptoms that were classified as pain disorder in DSM-IV. Evidence showing that psychological factors influence all types of pain (25) raises doubts about the validity and reliability of the distinctions between pain associated with psychological, medical or traumatic factors.

Most subjects with a DSM-IV diagnosis of pain disorder could be appropriately diagnosed as having DSM-V somatic symptom disorders, whereas others could meet the diagnosis of a new mental disorder defined as "psychological factors affecting other medical conditions".

Regardless of the diagnostic changes recently brought about by DSM-V, and despite the doubts regarding the validity of the diagnosis, neuroimaging studies have identified important components of the mental processes associated with a DSMIV diagnosis of pain disorder.

Functional magnetic resonance imaging studies have shown that patients with pain disrder and controls have different cerebral responses to pain stimuli: for example, the cerebral processing of noxious heat stimuli induces pain-related hypoactivation of the ventromedial prefrontal/orbitofrontal cortex, and hyperactivation of the parahippocampus, amygdala and anterior insula in patients with somatoform pain disorder (26). Furthermore, a negative emotional context such as sadness the same subjects contributes to greater sensitivity to low levels of pain probably due to the stronger activation of the anterior/posterior insula during low-pain stimuli (27). However, although neuroimaging studies provide information about the involvement of a specific neural system in pain disorder, the observed differences in neural activation between clinical and control groups cannot be interpreted univocally, but should be conceptualised by considering at least three different interpretations: as a cause of the illness, as an effect of the illness, or as compensation for the illness.

\section{REFERENCES}

1. Hiller W, Rief W, Brähler E. Somatization in the population: from mild bodily misperceptions to disabling symptoms. Soc Psychiatry Psychiatr Epidemiol. 2006; 41: 704-12.

2. Wessely S, Nimnuan C, Sharpe M. Functional somatic syndromes - one or many? Lancet. 1999; 354: 936-9.

3. American Psychiatric Association (APA). Diagnostic and statistical manual of mental disorders. Washington, DC: American Psychiatric Press. 1980.

4. Kroenke K. Patients presenting with somatic complaints: epidemiology, psychiatric comorbidity and management. Int J Methods Psychiatr Res. 2003; 12: 34-43.

5. Mayou R, Kirmayer LJ, Simon G, Kroenke K, Sharpe M. Somatoform disorders: time for a new approach in DSM-V. Am J Psychiatry. 2005; 162: 847-55.

6. Lee S. A Chinese perspective of somatoform disorders. J Psychosom Res. 1997; 43: 115-9.

7. American Psychiatric Association (APA). 
Diagnostic and statistical manual of mental disorders. 4th ed., Text Revised. Washington, DC: American Psychiatric Association. 2000.

8. Dimsdale J, Creed F, Escobar J, Sharpe M, Wulsin L, Barsky A, et al. Somatic symptom disorder: an important change in DSM. J Psychosom Res. 2013; 75: 223-8.

9. Dimsdale J, Sharma N, Sharpe M. What do physicians think of somatoform disorders? Psychosomatics. 2011; 52: 154-9.

10. Wittchen HU, Jacobi F. Size and burden of mental disorders in EuropeVa critical review and appraisal of 27 studies. Eur Neuropsychopharmacol. 2005; 15: 357-76.

11. Fink P, Sørensen L, Engberg M, Holm M, Munk-Jørgensen P. Somatization in primary care. Prevalence, health care utilization, and general practitioner recognition. Psychosomatics. 1999; 4: 330-8.

12. Escobar JI. Developing practical indexes of somatization for use in primary care. J Psychosom Res. 1997; 42: 323-8.

13. Rief W, Rojas G. Stability of somatoform symptoms-implications for classification. Psychosom Med. 2007; 69: 864-9.

14. Jackson JL, Passamonti M. The outcomes among patients presenting in primary care with a physical symptom at 5 years. J Gen Intern Med. 2005; 20: 1032-7.

15. Noyes R, Stuart SP, Watson DB. A reconceptualization of the somatoform disorders. Psychosomatics. 2008; 49: 14-22.

16. Chou R, Shekelle P. Will this patient develop persistent disabling low back pain? JAMA. 2010; 303: 1295-302.

17. Keijsers E, Feleus A, Miedema HS, Koes BW, Bierma-Zeinstra SMA. Psychosocial factors predicted nonrecovery in both specific and nonspecific diagnoses at arm, neck, and shoulder. J Clin Epidemiol. 2010; 63: 1370-9.

18. Hausteiner C, Bornschein S, Bubel E, et al.
Psychobehavioral predictors of somatoform disorders in patients with suspected allergies. Psychosom Med. 2009; 71: 1004-11.

19. Rief W, Mewes R, Martin A, Glaesmer H, Braehler E. Are psychological features useful in classifying patients with somatic symptoms? Psychosom Med. 2010; 72: 648-55.

20. Konnopka A, Schaefert R, Heinrich S, Kaufmann C, Luppa M, Herzog W, et al. Economics of medically unexplained symptoms: a systematic review of the literature. Psychother Psychosom. 2012; 81: 265-75.

21. Hiller W, Fichter MM. High utilisers of medical care: a critical subgroup among somatising patients. J Psychosom Res. 2004; 56: 437-43.

22. American Psychiatric Association (AMA). Diagnostic and statistical manual of mental disorders. 5th ed., DSM-5. Arlington, VA: American Psychiatric Publishing; 2013.

23. Hiller W, Heuser J, Fichter MM. The DSMIV nosology of chronic pain: a comparison of pain disorder and multiple somatization syndrome. Eur J Pain. 2000; 4: 45-5.

24. Aigner M, Bach M. Clinical utility of DSMIV pain disorder. Compr Psychiatry. 1999; 40: 353-7.

25. Rainville P, Bao QV, Chretien P. Pain-related emotions modulate experimental pain perception and autonomic responses. Pain. 2005; 118: 306-18.

26. Gündel H, Valet M, Sorg, C, Huber D, Zimmer $\mathrm{C}$, Sprenger T, et al. Altered cerebral response to noxious heat stimulation in patients with somatoform pain disorder. Pain. 2008; 137: 413-21.

27. Yoshino A, Okamoto Y, Yoshimura S, Shishida K, Toki S, Doi M, et al. Distinctive neural responses to pain stimuli during induced sadness in patients with somatoform pain disorder: an fMRI study. Neuroimage Clin. 2013; 2: 782-9. 